\title{
Development and Validation of a One-Step RT-qPCR Assay for Identifying Common Fusion Gene Transcripts Associated with the Prognosis of Mexican Children with B-Lineage Acute Lymphoblastic Leukemia
}

Juan Manuel Mejía-Aranguré ( $\nabla$ arangurejm@hotmail.com )

IMSS: Instituto Mexicano del Seguro Social https://orcid.org/0000-0003-0949-461X

Minerva Mata-Rocha

CONACYT: Consejo Nacional de Ciencia y Tecnologia

Angelica Rangel-López

IMSS: Instituto Mexicano del Seguro Social

Juan Carlos Núñez-Enríquez

IMSS: Instituto Mexicano del Seguro Social

Elva Jiménez-Hernández

IMSS: Instituto Mexicano del Seguro Social

Blanca Angélica Morales-Castillo

IMSS: Instituto Mexicano del Seguro Social

Norberto Sánchez-Escobar

UABJO Faculty of Medicine: Universidad Autonoma Benito Juarez de Oaxaca Facultad de Medicina

Omar Alejandro Sepúlveda-Robles

CONACYT: Consejo Nacional de Ciencia y Tecnologia

Juan Carlos Bravata-Alcántara

Hospital Juárez de México: Hospital Juarez de Mexico

Alan Steve Nájera-Cortés

Hospital Juárez de México: Hospital Juarez de Mexico

María Luisa Pérez-Saldivar

IMSS: Instituto Mexicano del Seguro Social

Janet Flores-Lujano

IMSS: Instituto Mexicano del Seguro Social

David Aldebarán Duarte-Rodríguez

IMSS: Instituto Mexicano del Seguro Social

Norma Angélica Oviedo de Anda

IMSS: Instituto Mexicano del Seguro Social 


\section{Carmen Alaez Verson}

INMEGEN: Instituto Nacional de Medicina Genomica

Jorge Alfonso Martín-Trejo

IMSS: Instituto Mexicano del Seguro Social

María de Los Ángeles Del Campo-Martínez

IMSS: Instituto Mexicano del Seguro Social

José Esteban Muñoz-Medina

IMSS: Instituto Mexicano del Seguro Social

César Raúl González-Bonilla

IMSS: Instituto Mexicano del Seguro Social

Maria de los Angeles Hernandez Cueto

IMSS: Instituto Mexicano del Seguro Social

Aurora Medina-Sansón

Hospital Infantil de México Federico Gomez: Hospital Infantil de Mexico Federico Gomez

Raquel Amador-Sánchez

IMSS: Instituto Mexicano del Seguro Social

José Gabriel Peñaloza-González

Hospital Juárez de México: Hospital Juarez de Mexico

José Refugio Torres-Nava

Moctezuma Pediatric Hospital: Hospital Pediatrico Moctezuma

Rosa Martha Espinosa-Elizondo

Hospital General de México Dr Eduardo Liceaga: Hospital General de Mexico Dr Eduardo Liceaga

Beatriz Cortés-Herrera

Hospital General de México Dr Eduardo Liceaga: Hospital General de Mexico Dr Eduardo Liceaga

Luz Victoria Flores-Villegas

ISSSTE: Instituto de Seguridad y Servicios Sociales de los Trabajadores del Estado

Laura Elizabeth Merino-Pasaye

ISSSTE: Instituto de Seguridad y Servicios Sociales de los Trabajadores del Estado

Karina Anastacia Solís-Labastida

IMSS: Instituto Mexicano del Seguro Social

Martha Margarita Velázquez-Aviña

Hospital Juárez de México: Hospital Juarez de Mexico

Jessica Denisse Santillán-Juárez

ISSSTE: Instituto de Seguridad y Servicios Sociales de los Trabajadores del Estado

Alma Gurrola-Silva

ISSSTE: Instituto de Seguridad y Servicios Sociales de los Trabajadores del Estado

Alejandra Jimena García-Velázquez

ISSSTE: Instituto de Seguridad y Servicios Sociales de los Trabajadores del Estado

Gabriela Alicia Hernández Echáurregui 
Moctezuma Pediatric Hospital: Hospital Pediatrico Moctezuma

\section{Haydeé Rosas-Vargas}

IMSS: Instituto Mexicano del Seguro Social

\section{Research Article}

Keywords: Fusion gene transcripts, Acute lymphoblastic leukemia, Prognosis, RT-qPCR Diagnostic, Molecular biomarkers, Therapeutic targets.

Posted Date: June 2nd, 2021

DOl: https://doi.org/10.21203/rs.3.rs-526521/v1

License: (c) (i) This work is licensed under a Creative Commons Attribution 4.0 International License. Read Full License 


\section{Abstract}

The aims of the present study were to optimize the detection of common fusion gene transcripts associated with childhood B-ALL prognosis through the development and validation of a one-step RTqPCR assay and to identify the prevalence of these genes in a cohort of pediatric patients attended in public hospitals in Mexico City. The one-step RT-qPCR assay was sensitive, specific, easy to use, fast and cost efficient. The standard curves were linear, with amplification efficiencies between $97-107 \%$; coefficients of variation were $2.1-3.0 \%$ in reproducibility and $2.6-3.8 \%$ in repeatability, with agreement between two different laboratories. The prevalence of fusion gene transcripts was $E T V 6-R U N X 1,10.3 \%$; TCF3-PBX1, 7.5\%; BCR-ABL1 $1 p^{190}, 3.6 \%$; and KMT2A-AFF1, 2.8\%. The prevalence of the ETV6-RUNX1 fusion was low, consistent with the findings in Hispanics. The KMT2A-AFF1 fusion was not exclusively present in infants, as we also found it in patients 4-16 years of age, in whom it was related to a poor prognosis. TCF3-PBX1 patients were associated with an intermediate outcome. The present study highlights the importance of the detection of common genetic fusions in Mexican children with ALL, taking into account that some have molecular subtypes associated with a poor prognosis.

\section{Introduction}

B-lineage acute lymphoblastic leukemia (B-ALL) is a malignant transformation of B-lymphoid cell precursors characterized by uncontrolled proliferation and accumulation of leukemia blasts in the bone marrow [1]. B-ALL is the most frequent malignancy diagnosed in children and adolescents worldwide [2]. In the Mexican population, it is the second leading cause of death in the group aged between 5-14 years, with an estimated incidence of 49.5 cases per million children under 15 years of age [3]. Previous studies conducted in Mexico City have pointed out the importance of continuing with the refinement of diagnosis and prognostic stratification schemes to increase the survival rates in our population $[4,5]$. Fusion gene transcript detection has contributed to improved survival rates in children from other populations, mainly in developed countries [6].

The presence of translocations in cancer reflects the genomic instability of cells and may characterize specific pathways involved in malignancy; however, the mechanisms that cause these translocations are poorly understood. To date, some mechanisms have been proposed to explain chromosomal rearrangements, such as illegitimate $\mathrm{V}(\mathrm{D}) \mathrm{J}$ recombinase activity or switch recombination, as well as nonhomologous end joining (NHEJ) after double-stranded break DNA (DSB) occurs on two different chromosomes [7, 8]. Translocations, often associated with hematological malignancy, have been postulated as one of the main causes of oncogenic transformations associated with leukemogenesis during fetal development $[9,10]$. However, they are not sufficient by themselves to trigger the leukemic process. Additional cooperating mutations are usually required $[1,11]$. Different studies have reported the detection of $t(8 ; 21)$ and $t(12 ; 21)$ translocations in newborns at a rate of $\sim 1 \%$. These translocations have been associated with an increase in the risk of developing B-ALL $[11,12]$. 
Genes involved in translocations that fuse to the preferred partner or other less frequent genes may result in the deregulation of one of the genes involved (underexpression of proto-oncogenes or inhibition of the tumor suppressor gene) or in the expression of oncogenic proteins such as $B C R / A B L 1$ [9]. The study of these translocations has allowed the characterization of four common subtypes in children with B-ALL: $\mathrm{t}$ $(4 ; 11)$ KMT2A-AFF1, $\mathrm{t}(12 ; 21)$ ETV6-RUNX1, and $\mathrm{t}(1 ; 19)$ TCF3-PBX1, which alter gene expression, and $\mathrm{t}$ $(9 ; 22) B C R-A B L 1$, which disturbs signaling pathways.

For these common subtypes, the $B C R / A B L 1$ fusion gene produces a hybrid transcript that encodes a protein with tyrosine kinase activity leading to transformation. Both MLL (KMT2A) and ETV6 (TEL) have been labeled "promiscuous" genes because they are rearranged with more than 80 and 160 different partner genes, respectively [13]. MLL (KMT2A) is mainly rearranged with AF4 in B-ALL pediatric patients. KMT2A-AFF1 fusion is associated with childhood de novo leukemias originating in utero, and patients tend to have a poor prognosis. Another translocation encoding a protein with transformative properties is $\mathrm{t}(1 ; 19)$ (q23;p13), creating a TCF3-PBX1 fusion associated with the pre-B immunophenotype mainly associated with isolated central nervous system relapse that is present in approximately $5 \%$ of children with B-ALL [14].

Therefore, accurate detection of fusion genes in patients with B-ALL has prognostic implications favoring chemotherapy decisions, improving the therapeutic effect in terms of decreasing treatment-related toxicity and improving patient survival. Despite its undeniable importance, in Mexico City, detecting a wide range of fusion genes that may be associated with B-ALL prognosis can be complicated in terms of time and resources. Various molecular biology and cytogenetic techniques, such as banding analysis (karyotyping), fluorescent in situ hybridization (FISH), reverse transcription polymerase chain reaction (RTPCR) and, more recently, second-generation sequencing techniques [15], have been used for fusion gene transcript detection.

Real-time qPCR has facilitated the diagnosis of a variety of fusion genes because it can quantify and control very small amounts of nucleic acids while amplification occurs. The primary objective of this study was to optimize the detection of rearrangements associated with B-ALL prognosis using one-step RT-qPCR in which the synthesis of cDNA and qPCR are performed in a single reaction, improving the sensitivity and the time for obtaining valid results to timely diagnose and stratify a patient with leukemia. In the present study, it was also possible to identify the prevalence of the most common fusion gene transcripts associated with the prognosis of B-ALL in a cohort of pediatric patients attended in public hospitals in Mexico City over a two-year period.

\section{Methodology}

We conducted the present study in two stages: A) development of the method and B) analysis of clinical samples. An overview of the research described here is presented in Fig. 1.

A) Development of the method 


\subsection{Primer and probe design}

RT-qPCR for each fusion gene transcript that is clinically relevant in pediatric leukemia (ETV6-RUNX1, TCF3-PBX1, BCR-ABL1P190, KMT2A-AFF1) and an endogenous control transcript (GAPDH) were performed using specific primers and TaqMan probes. Gene sequences of each fusion transcript and $G A P D H$ were obtained from the National Center for Biotechnology Information (https://www.ncbi.nlm.nih.gov/) for alignment and design procedures. The specific primers and probes were designed using the website Primer3Plus software program, and a BLAST search was performed to ensure specificity to the fusion target region. The melting temperature $(\mathrm{Tm})$ should be $60 \pm 3^{\circ} \mathrm{C}$ and $70 \pm$ $3^{\circ} \mathrm{C}$ for primers and probes, respectively, and hairpin formation should not occur. Expected amplicons should be between $80-150 \mathrm{pb}$. The position of the primers is shown in Supplementary Fig. 1. At this moment, the sequence of primers and probes is protected for the reason of a patent claim.

\subsection{Positive RNA controls}

To evaluate the primer sets, we used total RNAs derived from Reh (ATCC $\AA$ CRL-8286 ${ }^{\text {TM }}$ ), SUP-B15 (ATCC $\circledast$ CRL-1929 ${ }^{\text {TM }}$ ) and RS4;11 (ATCC $®$ CRL-1873 ${ }^{\text {TM }}$ ) leukemia cell lines for ETV-6-RUNX1, BCR-ABL1, and KMT2A-AFF1 fusion, respectively. For TCF3-PBX1 fusion, we used RNA derived at diagnosis from a positive patient (P300). Cell lines were maintained in RPMI-1640 medium (Gibco BRL) or Dulbecco's medium supplemented with $10 \%$ fetal calf serum, $1 \mathrm{nM}$ glutamine, and $50 \mathrm{mg}$ penicillin/streptomycin in a saturated humidified atmosphere with $5 \% \mathrm{CO}_{2}$ at $37^{\circ} \mathrm{C}$.

\subsection{Selection of optimal one-step RT-qPCR TaqMan system for testing fusion genes}

To select an appropriate real-time one-step RT-qPCR kit for this standardization, we compared the efficiency of different commercially available technologies: a) QuantiTect Probe RT-PCR (Qiagen, Venlo, Netherlands), b) AgPath-ID One-Step RT-PCR Reagents (Thermo Fisher Scientific), c) Superscript III OneStep RT-PCR (Invitrogen), and d) FastGene ${ }^{\circledR}$ PROBE One Step (NIPPON Genetics Europe, Germany). Each method was performed according to the manufacturer's instructions on a StepOnePlus ${ }^{\mathrm{TM}}$ Real-Time PCR System (Applied Biosystems, Foster City, CA) using positive RNA controls, probes and primers.

\subsection{Primer, probe and total RNA concentration}

As total RNA was added directly to the RT-qPCR mixture, we determined the maximum (optimal) amount of total RNA that could be added without an inhibitory effect. We used different concentrations of positive RNA controls, which were diluted in series to obtain the following final amounts: $50,100,200$, 300 , and $500 \mathrm{ng} /$ reaction. Different gradients of temperature alignment $\left(56-64^{\circ} \mathrm{C}\right)$, primer concentrations $(200,250$ and $300 \mathrm{nM})$, and probe concentrations $(50,125,250$ and $500 \mathrm{nM})$ were used to optimize PCR conditions for each common fusion gene.

\subsection{Cloning of fusion gene transcripts, preparation of standard curves and efficiency determination}


Plasmids containing the GAPHD, ETV6-RUNX1, KMT2A-AFF1, BCR-ABL $1 p^{190}$ and TCF3-PBX1 CDNA transcripts were generated by cloning the PCR products amplified from RNA extracted from cell lines and one patient (TCF3-PBX1), as described in Pakakasama et al. (2008) [16], into the pJET1.2 vector using the CloneJET ${ }^{T M}$ PCR Cloning Kit (Thermo Scientific ${ }^{T M}$ ). Then, plasmids were transformed and propagated in Escherichia coli JM109, extracted using the GeneJET Plasmid Miniprep Kit (Thermo Scientific ${ }^{T M}$ ) and sequenced using an ABI3500xL genetic analyzer (Applied Biosystems, Waltham, MA, USA). Plasmid DNA concentration was calculated using a Qubit dsDNA BR assay (Life Technologies Co., Carlsbad, CA, USA) to improve the accuracy of quantification. We converted DNA plasmid size to copy numbers by multiplying the number of base pairs of each plasmid (ETV-6-RUNX1, BCR-ABL 1, KMT2A-AFF1, TCF3$P B X 1)$ by the average molecular mass of one base pair $(\sim 660 \mathrm{~g} / \mathrm{mol})$ to obtain the approximate mass and then divided by Avogadro's constant: $\mathrm{N}_{A}=6.022 \times 10^{23}$ molecules. The plasmids were serially diluted as follows: $1.0 \times 10^{2}, 1.0 \times 10^{3}, 1.0 \times 10^{4}, 1.0 \times 10^{5}$, and $1.0 \times 10^{6}$ copies/reaction with each dilution, in quadruplicate. Linear regression was performed between the cycle threshold value (Ct-value) and the $\log 10$ of the copy number. The amplification efficiency for each fusion reaction was calculated using the equation $E=10(-1 /$ slope $)$ and converted to $E \%$ by $(E-1) \times 100$.

\subsection{Efficiency and specificity assays}

Plasmids with the TCF3-PBX1, ETV6-RUNX1, BCR-ABL1190, KMT2A-AFF1, EP300-ZNF384, CREBBPSRGAP2B, DNAH14-IKZF1, ETV6-SNUPN, and ETV6-NUFIP1 fusion genes were used to test the existence of cross-reactions. A mixture of all plasmids was prepared at 300,000 copies/reaction and tested for each TCF3-PBX1, ETV6-RUNX1, BCR-AB1P ${ }^{190}$, and KMT2A-AFF1 fusion in one real-time PCR protocol, including the positive RNA and water controls. Additionally, a set of total RNA samples of clinical isolates with positive and negative results for different fusion genes were used.

\subsection{Blind comparisons using QuanDx}

The reliability and sensitivity of the one-step RT-qPCR assay developed in this study was compared with the QuanDx Kit, a qualitative in vitro diagnostic test for the screening of 30 fusion genes involved in chronic and acute leukemia, at a separate laboratory (Hospital Juarez de Mexico). Forty-five clinical samples were blindly analyzed at that hospital using the QuanDX Kit.

\subsection{Repeatability and reproducibility}

To evaluate the repeatability and reproducibility of the one-step RT-qPCR assay, we used positive RNA controls for each transcript. Regarding the assessment of repeatability, the detection of each transcript was independently tested four times on five days by the same operator, while for the reproducibility, each fusion was analyzed four times by three different operators on three different days for all fusion genes and the internal control GAPDH.

B) Analysis of clinical samples

\subsection{Patients}


This study was conducted from January 2018 to December 2019 and included bone marrow available samples obtained from 252 patients below the age of 19 years newly diagnosed with ALL from eight public hospitals in Mexico City. The study was approved by the National Ethics and Scientific Committees (R-2015-785-121). Written informed consent was obtained from the child's parents to participate in the study. The diagnosis of leukemia was based on the morphology of leukemic cells and immunophenotyping. Table 2 contains relevant information of patients.

\subsection{RNA isolation from bone marrow samples}

Total RNA was extracted from white blood cells (WBCs) of bone marrow using Direct-zol MiniPrep (Zymo Research, USA) according to the manufacturer's protocol. The RNA was suspended in a final volume of $30 \mu \mathrm{l}$ elution buffer and stored at $-80^{\circ} \mathrm{C}$ until use. Concentrations of RNAs were measured using a Nanodrop ND-1000 spectrophotometer (Thermo Fisher Scientific, Wilmington, MA, USA).

\subsection{Clinical samples: the quality of total RNA}

The quality of RNA was examined using $100-150$ ng of total RNA by analysis of glyceraldehyde-3'phosphate dehydrogenase (GAPDH) transcript copy numbers determined by plasmid DNA standard curves. The copy number needed to be greater than $2.5 \times 10^{5}$, which is considered a suitable RNA quantity for RT-qPCR screening purposes, as has been previously reported [17]. Only samples and RNA cell lines with sufficient quality were analyzed.

\subsection{Reverse transcription-polymerase chain reaction (RT-PCR) for fusion gene detection}

The reproducibility and repeatability tests were carried out with the QuantiTect Probe RT-PCR Kit with $5 \mu$ l of 2x QuantiTect Probe RT-PCR Master Mix containing ROX ${ }^{\mathrm{TM}}$ as passive reference dye and HotStarTaq ${ }^{\circledR}$ DNA Polymerase, $0.1 \mu \mathrm{l}$ QuantiTect RT Mix, $200 \mathrm{nM}$ each primer, and $500 \mathrm{nM}$ each TaqMan probe in a final volume of $50 \mu \mathrm{l}$. Thermal cycling conditions were as follows: $30 \mathrm{~min}$ at $50^{\circ} \mathrm{C}, 15 \mathrm{~min}$ at $95^{\circ} \mathrm{C}$, followed by $38 \mathrm{cycles}$ of $94^{\circ} \mathrm{C}$ for $15 \mathrm{sec}$ and $60^{\circ} \mathrm{C}$ for $1 \mathrm{~min}$. Amplification reactions contained $50-100$ ng of the total RNA. GAPDH was used as an internal gene control for each sample.

\subsection{Statistical analysis}

Statistical analyses were performed using SPSS IBM (Statistical Package for the Social Sciences, Inc., Version 21, Chicago, IL, USA. [18] The prevalence of the four most frequent gene rearrangements in pediatric ALL was obtained. The Kaplan-Meier method was used to estimate overall survival (OS) and disease-free survival (DFS) rates. The log-rank test was used to compare the survival curves at the $<0.05$ level of significance.

\section{Results}

\subsection{Application of one-step RT-qPCR TaqMan for fusion gene transcript detection}


The first step in this study consisted of the development of a combination of primers and TaqMan probes for the detection of the fusion gene transcripts ETV6-RUNX1, KMT2A-AFF1, BCR-ABL1P190 and TCF3$P B X 1$. One-step RT-qPCR for detecting these alterations was used in the present study. It is considered an advantageous method because it is less expensive, reduces process time, and requires limited handling of samples, thus reducing pipetting errors, cross contamination between RT and PCR steps, and other sources of error. We tested the predesigned probe and oligonucleotides with different kits, and we observed differences in Ct values among different kits. With the QuantiTect Probe (Qiagen) kit, the best results were obtained with Ct between 16.7 and 27.5, while the other kits were greater than Ct 21.8 (Supplementary Fig. 2). Therefore, we proceeded to use the QuantiTect probe kit for the following tests to standardize the detection of fusion gene transcripts. Additionally, the Positive RNA controls showed satisfactory results using a QuantiTect probe kit (Table 1).

\subsection{Optimization of one step RT-qPCR}

To optimize the one-step RT-qPCR reaction, we performed a temperature gradient experiment using control positive RNA that was subjected to 6 different annealing temperatures $\left(58^{\circ} \mathrm{C}-64^{\circ} \mathrm{C}\right)$. We found that the optimal annealing temperature was $60^{\circ} \mathrm{C}$ for detection of all fusion transcripts, and no amplification product could be observed above $62^{\circ}$. Additionally, the optimal concentration of the different primer and TaqMan probe sets used was determined. We found significant differences among the various primers, but not among probe concentrations. Based on the results, a final concentration of TaqMan probes of $0.5 \mu \mathrm{mol} / \mathrm{l}$ was selected for detection of all fusion gene transcripts. The highest detection was obtained at a primer concentration of $0.4 \mu \mathrm{mol} / \mathrm{l}$ for all.

In addition, we were able to detect fusion genes using only 50-100 ng of total RNA with good analytical sensitivity for detecting $\geq 1000$ copies/ $\mu$ l of transcripts using a different set of primers and probes for each fusion gene. Otherwise, the problems related to the presence of gDNA, degradation of RNA and contaminants that can inhibit RT or PCR were solved by isolation of RNA using column-based purification protocols and DNAse treatment (Direct-zol ${ }^{\mathrm{TM}} \mathrm{RNAMiniprep,} \mathrm{Zymo} \mathrm{Research,} \mathrm{California,} \mathrm{USA)} \mathrm{compared} \mathrm{to}$ other methods, such as RNAzol® RT (Sigma-Aldrich, Missouri, USA) or TRIzol® Reagent (Thermo Fisher Scientific, Massachusetts, USA) (data not shown).

\subsection{PCR primers and probe design resulted in good amplification efficiency and specificity.}

Construction of plasmids was used to obtain standard reference curves to infer the levels of transcripts of interest from clinical samples or cell lines (Fig. 2). GAPDH mRNA was used as an internal control for the evaluation of RNA integrity. In the GAPDH calibration curve, the $\mathrm{Ct}$ was linearly proportional to the logarithm of the number of input copies by at least five orders of magnitude $(R 2=0.9973, E \%=107$, Fig. 2E). To be considered adequate, the $\mathrm{Ct}$ values of the GAPDH signal must be less than 20 , while $\mathrm{Ct}$ values above 20 may not be related to the quantification and quality of good RNA (Table 1). The amplification efficiencies obtained were $101 \%$ for ETV6-RUNX1, 97\% for KMT2A-AFF1, 98\% for BCRABL 1p190 and $106 \%$ for TCF3-PBX1. Efficiency was calculated using primer and probe sets considering a 
slope (S) of the linear regression curve indicating an optimal PCR in which the number of copies was duplicated in each cycle. Typical calibration and amplification curves are shown in Fig. 2 (A-E).

Assay specificity was evaluated by testing RNA from the bone marrow of patients without leukemia but with various hematological diseases (e.g., pancytopenia, chronic myeloid leukemia, idiopathic thrombocytopenic purpura) and from plasmid mixtures. The results were negative for the detection of fusion gene transcripts (Ct value: 40 ), indicating the absence of nonspecific amplifications related to the detection of spurious sequences.

Additionally, in parallel with our one-step RT-qPCR method, independent testing of 45 samples was conducted in an external laboratory (Hospital Juarez de Mexico1) using a QuanDX kit. The results showed $100 \%$ concordance between the two laboratories when the four fusion genes of interest were analyzed (Supplementary Table 1). Out of the 45 samples analyzed, only in $7(15.5 \%)$ was a fusion gene transcript detected. Two were positive for ETV6-RUNX1 (4.4\%), three were positive for TCF3-PBX1 (6.6\%) and two were positive for BCR-ABL $1 p 190$ (4.4\%). One sample tested with the QuanDX kit was positive for another MLL-ENL fusion gene (sample 20), and another sample showed RUNX1/CBFA2T3 fusion (sample 1) in addition to ETV6-RUNX1 (both fusion genes were not included in the one-step RT-qPCR) (Supplementary Table 1).

\subsection{Reproducibility and repeatability of real-time RT-PCR.}

To assess interlaboratory variation, positive RNA controls were tested by three independent analysts at different time periods. The $\mathrm{Ct}$ values for all the fusion genes are listed in Table 1. We obtained good precision, and the coefficients of variation (CV) of reproducibility and repeatability were $2.1-3.0 \%$ and $2.6-3.8 \%$, respectively (Table 1 ).

Table 1

Precision of OneStep RT-PCR Taqman Assay

\begin{tabular}{|llllllll|}
\hline Assay & & Reproducibility & & Repeatability & \\
\hline Cell line & Target & Mean (range) & SD & CV (\%) & Mean (range) & SD & CV (\%) \\
REH & GAPDH & $17.1(16.1-17.9)$ & 0.4 & 2.6 & $16.9(16.0-17.9)$ & 0.5 & 3.0 \\
& TEL-RUNX1 & $26.4(25.6-27.7)$ & 0.6 & 2.3 & $26.3(25.0-27.7)$ & 0.7 & 2.6 \\
P300 & GAPDH & $19(18.4-19.6)$ & 0.4 & 2.1 & $18.5(17.4-19.4)$ & 0.6 & 3.5 \\
& E2A-PBX1 & $26.5(25.6-27.9)$ & 0.7 & 2.6 & $26.3(25.0-27.9)$ & 0.8 & 2.9 \\
RS4 & GAPDH & $17.4(16.7-18.1)$ & 0.5 & 2.7 & $17.3(16.0-18.1)$ & 0.5 & 3.1 \\
& KMT2-AFF1 & $26.4(25.4-27.5)$ & 0.6 & 2.4 & $26.9(26.0-28.4)$ & 0.7 & 2.7 \\
SUP- & GAPDH & $17.0(16.0-17.9)$ & 0.5 & 3.0 & $16.7(15.3-17.8)$ & 0.6 & 3.8 \\
\cline { 2 - 7 } & $\begin{array}{l}\text { BCR-ABL1 } \\
\text { P190 }\end{array}$ & $26.1(25.1-27.1)$ & 0.6 & 2.4 & $26.0(24.4-27.0)$ & 0.7 & 2.9 \\
\cline { 2 - 7 } & & & & & & & \\
\hline
\end{tabular}


Table 1. The results shown are the $\mathrm{Ct}$ means (ranges) of the independent determinations, with the standard deviation (SD) and coefficient of variation (CV) shown. $\left(^{*}\right)$ Independent assays were performed four times on five different days for repeatability and $(* \star)$ four times on three days for reproducibility.

\subsection{Prevalence and prognostic impact of fusion gene transcripts}

Samples from 253 children with newly diagnosed ALL were analyzed. Samples with good quality for analysis were considered. A total of 136 boys (52.1\%) were included with a mean age of 8.6 years (range, 0.2-17.7 years). The mean follow-up time was 398 days ( $95 \% \mathrm{Cl}$ : $378-419$ days) for disease-free survival (DFS) and 404 days (Cl: 383-425 days) for overall survival (OS). During the follow-up, 17 (6.7\%) patients died, and $12(4.7 \%)$ relapsed (Table 2). Seven patients had Down's syndrome, one of whom had the transcript $B C R-A B L 1 p^{190}$. Of the samples analyzed by one-step RT-qPCR, $75.9 \%$ were negative for the four fusion genes analyzed, while only $24.1 \%(61 / 253)$ were positive. The prevalence of fusion gene transcripts was as follows: ETV6-RUNX1 (10.3\%), TCF3-PBX1 (7.5\%), KMT2A-AFF1 (2.8\%) and BCR$\operatorname{ABL} 1 p^{190}(3.6 \%)$ (Table 2). 
Table 2

Clinical features of patients with ALL by molecular subtype

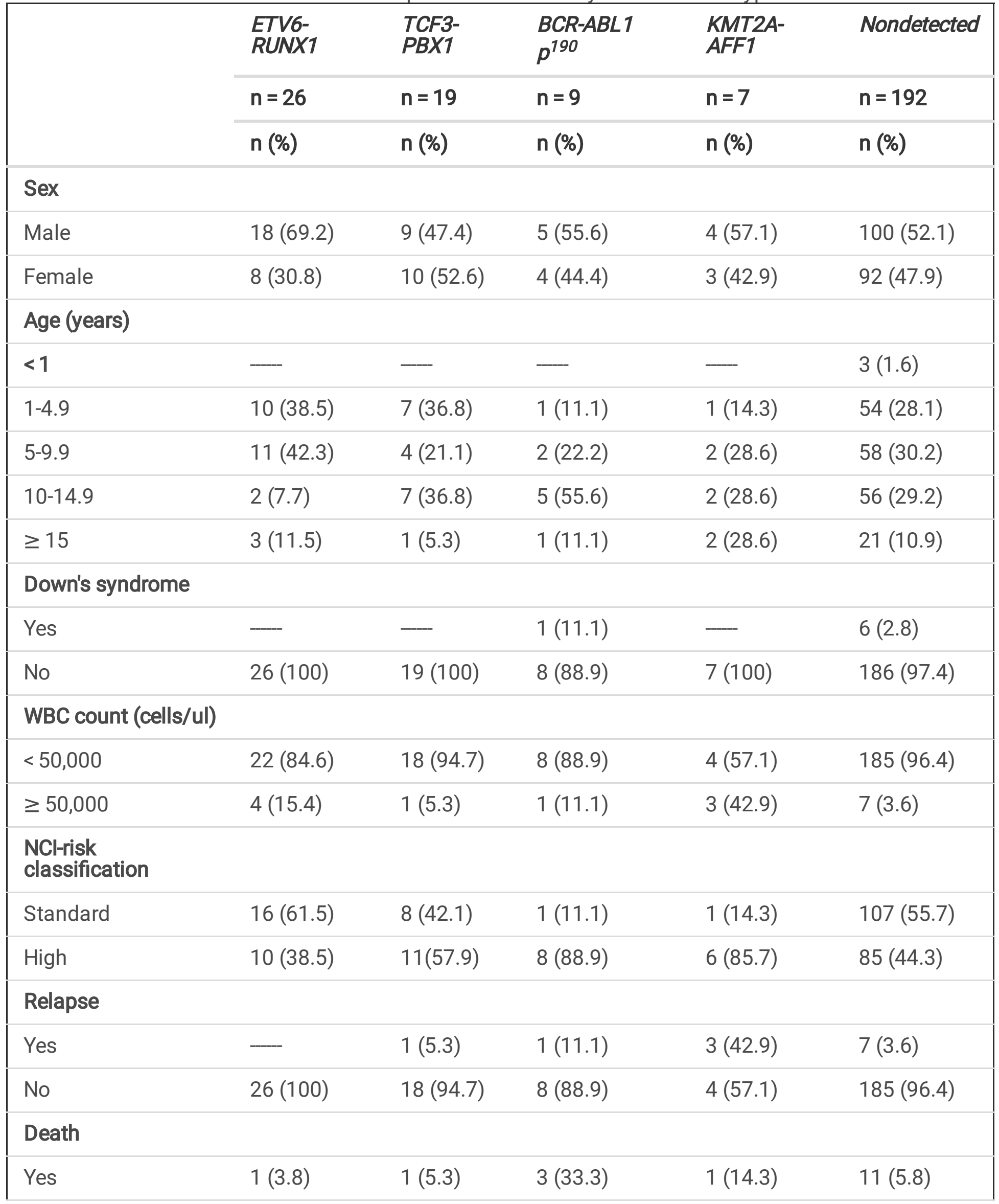




\begin{tabular}{|llllll|}
\hline & $\begin{array}{l}\text { ETV6- } \\
\text { RUNX1 }\end{array}$ & $\begin{array}{l}\text { TCF3- } \\
\text { PBX1 }\end{array}$ & $\begin{array}{l}\text { BCR-ABL1 } \\
\boldsymbol{p}^{190}\end{array}$ & $\begin{array}{l}\text { KMT2A- } \\
\text { AFF1 }\end{array}$ & Nondetected \\
\cline { 2 - 6 } & $\mathrm{n}=26$ & $\mathrm{n}=19$ & $\mathrm{n}=9$ & $\mathrm{n}=7$ & $\mathrm{n}=192$ \\
\hline $\mathrm{n}(\%)$ & $\mathrm{n}(\%)$ & $\mathrm{n}(\%)$ & $\mathrm{n}(\%)$ & $\mathrm{n}(\%)$ \\
\hline No & $25(96.2)$ & $18(94.7)$ & $6(66.7)$ & $6(85.7)$ & $181(94.2)$ \\
\hline
\end{tabular}

Kaplan-Meier estimations for OS and DFS by considering each fusion gene transcript are shown in Fig. 3 . As indicated, there were no significant differences in OS; in contrast, the differences in DFS were statistically significant among the fusion genes $(p=0.02)$.

ETV6-RUNX1 was the most common fusion gene transcript (10.3\%), present in all age groups, mainly between 1.0 and 10 years. Approximately $84.6 \%$ of these patients had a white blood cell count below 50,000 , and $61.5 \%$ were classified as standard risk. In this study, almost all patients with this fusion gene transcript remained in first complete remission at the time of conclusion of this study; only one exhibited a poor response and died (Table 2, Fig. 3). It therefore had a favorable impact on DFS and OS $(100 \%$ and $96 \%$, respectively). The least prevalent fusion gene transcript was $K M T 2 A-A F F 1$, observed in $2.8 \%$ of patients aged 1-16 years at diagnosis. These patients showed a higher overall white blood cell count (> $50,000,42.9 \%)$ at the time of diagnosis. Approximately $85.7 \%$ of these were classified as high risk, three relapsed, and one died. KMT2A-AFF1 patients displayed low DFS and OS rates (56\% and $80 \%$, respectively). On the other hand, the $t(1 ; 19)$ translocation (q23;p13) causing TCF3-PBX1 was present in nineteen patients (7.5\%) aged $1-16$ years. Of these, 94.7 had a white blood cell count below 50,000 at the time of diagnosis. Eleven (57.9\%) were classified as high risk, one relapsed and one patient died. In these patients, this translocation had a favorable impact on DFS and OS ( $89 \%$ and $88 \%$, respectively). Finally, transcribed $B C R-A B L 1 p^{190}$ showed a positivity rate of approximately $3.6 \%$, and most patients achieved complete remission. One relapse and three deaths occurred within this molecular subgroup. OS was $63 \%$ while the DFS was $86 \%$.

\section{Discussion}

In children with ALL attended at public hospitals in Mexico City, the identification of common fusion gene translocations associated with the prognosis of the disease is not routinely performed, but is available through research projects such as the present study. The purpose of this study was to identify the prevalence of ETV6-RUNX1, KMT2A-AFF1, TCF3-PBX1, and BCR-ABL1P190 fusion genes using a one-step TaqMan RT-qPCR assay and present the technical details of this assay. In Mexico, as in other countries, several groups have published different protocols for the detection of leukemia-specific fusion transcripts using conventional RT-PCR, and valuable prognostic information on human leukemias has been provided $[16,19-21]$. To improve the specificity and timeliness of the detection of common fusion gene transcripts, the abovementioned assay was developed and validated in the present study. All one-step RTqPCR assays for each fusion gene showed good sensitivities and specificities. The sensitivity could be 
explained, at least in part, by the elimination of the cDNA synthesis step using reverse transcription prior to PCR. Currently, a different enzyme with a higher efficiency is available in one-step kits. In our work, we compared different one-step kits and found that QuantiTect Probe RT-PCR (QIAGEN) showed high sensitivity for detecting the four fusion genes assessed.

Another important aspect of the one-step RT-qPCR assay was the precision obtained. It was estimated by the coefficient of variation, which reflects the day-to-day differences and variations of operators with different levels of experience. This coefficient was acceptably low to propose the use of this assay. Our results also showed high repeatability and reproducibility, and all cross-reactivity assays demonstrated that the primers and probes were specific for each fusion gene. The results of the interlaboratory comparison showed an acceptable concordance of results.

While commercially available multiple fusion gene transcript detection kits are relatively expensive, when considering the importance of detecting the four most common fusion gene transcripts associated with prognosis, the developed one-step real-time RT-PCR assay may be more cost-effective than QuanDx. This is supported by the fact that major problems for the determination of common fusion genes in Mexican health institutions are the lack of resources and specialized personnel.

\section{Prevalence and prognostic impact of common fusion genes}

It is well recognized that the B-lineage ALL (B-ALL) subtype has large genetic heterogeneity. Of importance, the fusion gene transcripts evaluated here are relevant for stratifying the risk of children with this disease (Table 2, Supplementary Fig. 3).

The frequencies of ETV6-RUNX1, TCF3-PBX1, KMT2A-AFF1 and BCR-ABL1p190 were $10.3 \%, 7.5 \%, 2.8 \%$ and $3.6 \%$, respectively, similar to a previous study conducted by our research group where a comparable number of clinical samples were analyzed [22]. Of note, the prevalence of these four fusion gene transcripts was $24.2 \%$, which is similar to the $20 \%$ detection reported in a recent study carried out in southern Mexico using an in vitro diagnostic test (HemaVision-Q28, DNA Diagnostic) [23]. Nonetheless, it has a low prevalence compared with studies conducted in other countries, particularly developed nations $[24,25]$. This highlights the importance of discovering new molecular determinants possibly contributing to the high incidence and mortality of ALL in the Mexican population.

\section{ETV6-RUNX1}

ETV6-RUNX1 is the most prevalent fusion gene transcript reported in children with ALL from developed countries ( $25 \%$ ), and it is associated with a favorable prognosis of the disease, currently reaching survival rates higher than $90 \%$ at 5 years of follow-up [26]. However, in the present study, the analysis revealed that 26 of 253 cases (10.3\%) presented the ETV6-RUNX1 fusion; most were male (69.2\%), with a $<50,000$ WBC count (84.6\%) in peripheral blood at diagnosis. It was present at all ages but most commonly observed in children under 10 years of age (Table 2). The low prevalence of ETV6-RUNX1 is consistent with previous Mexican reports using different detection methodologies: 8.7\% [27], 8.5\% [28], 
7.4\% [22], 6.9\% [23], 13.5\% [19], and 14.9\% [29]. These results are similar to those reported in Hispanics living in other countries $4.5 \%$ in Guatemalan and $14 \%$ in Hispanic residing in the Northern California [30, 31]. This finding is probably due to the genetic background of these populations [32].

However, it also raises concerns about whether this finding is due to a misdiagnosis. In this regard, we used RT-qPCR, which is considered a highly sensitive method to detect fusion gene transcripts. In addition, it is well known that fusion genes may have different breakpoints, making them undetectable using standard methods; however, this phenomenon occurs infrequently. Notably, the full identification of fusion gene transcripts is possible using next-generation sequencing (NGS) technology [33]. In this context, in a previous study by our research group using RNA-Seq analysis of 24 bone marrow samples of children with ALL, only one patient was ETV6-RUNX1 positive, which was consistent with the prevalence found in the present study[34]. Conversely, we found a novel fusion gene, ETV6-NUFIP1 and ETV6-SNUPN, that interrupts the ETV6 gene with break sites in the first and second exons, respectively; therefore, it is possible that these novel fusions play a causal role in tumorigenesis [34]. Nevertheless, although the prevalence of genetic fusion in the Mexican population is low, several groups have reported other genetic alterations related to $E T V 6$ and $A M L 1$ genes, such as deletions or duplications. Perez-Vera, 2005 reported $35 \%$ alterations in AML 1 copy numbers in LLA, and structural changes in ETV6 increased the number of ETV6 and $A M L 1$ consequences of polysomy of chromosomes 12 and 21, which play significant roles in disease progression [28].

\section{TCF3-PBX1}

The TCF3-PBX1 $\mathrm{t}(1 ; 19)$ (q23;pl3.3) translocation results in the expression of a protein that combines the transactivation domains of TCF3 with the DNA binding homeodomain region of $P B X 1$ [35]. TCF3-PBX1 fusion can promote cell transformation both in vitro and in vivo. Interestingly, the existence of a loss-offunction allele of TCF3 as a consequence of translocations in this gene has also been proposed. It reduces the levels of wild-type TCF3, which contributes to the development of leukemia [36]. Because TCF3 plays an important role as a mediator of B cell differentiation, TCF3 functions as a negative regulator of tumorigenesis [37]. The prevalence reported for TCF3-PBX1 fusion gene transcripts varies between 3-5\% in childhood ALL; however, in this study, the prevalence was $7.5 \%$ (19 of 253 patients). TCF3-PBX1 patients were distributed across all age groups, particularly in children with $<50,000$ WBCs at diagnosis. One child relapsed, and another died early during treatment (16.2 and 17.7 months after diagnosis, respectively). TCF3-PBX1-positive ALL has been related to an intermediate disease prognosis $[29,38]$. Nonetheless, currently, with the increase in the intensity of the subgroup of E2A-PBX1-positive patients, they have a similar survival as those patients classified as standard risk [39]. In the present study, we observed a DFS of $89 \%$ in patients with TCF3-PBX1, which correlates with previous reports in children with ALL and TCF3-PBX1 positivity. Importantly, in public hospitals in Mexico City, the TCF3$P B X 1$ group showed significant improvements in survival rates over time (data in preparation to publish) after the detection of this rearrangement through research project funding. In particular, the adverse prognostic value of these rearrangements has been overcome by using contemporary treatments such as Berlin-Frankfurt-Münster (BFM) [40, 41] and CCLG-ALL2008, a risk-based intensified treatment trial [42]. 
$B C R-A B L 1 p^{190}$

The $t(9 ; 22)(q 34 ; q 11)$ reciprocal translocation encodes a tyrosine kinase and is considered a pathogenetic driver that can be therapeutically targeted. There are several breakpoints in BCR that produce distinct BCR-ABL1 isoforms in children with ALL. In patients with chronic myeloid leukemia, the most common is the BCR-ABL1 $p^{220}$ isoform, while in the ALL subtype, it is the $p^{190}$ isoform associated

with a dismal prognosis [43]. However, treatment with tyrosine kinase (TK) inhibitors has improved the overall survival of patients with this rearrangement [18]. In the present study, the BCR-ABL1p ${ }^{190}$ translocation was identified in $3.6 \%$ of patients, with the majority being over 10 years of age at the time of diagnosis confirmation, which is similar to that reported in other populations. The survival analysis showed low survival rates (DFS: $86 \%$ and OS: $63 \%$ ) in patients with this translocation, but they are similar to those reported in other studies [44]. Several mechanisms have been related to relapse and death among patients with $B C R-A B L 1$. Among these mechanisms is resistance to imatinib due to mutations within the ABL1 (tyrosine kinase) domain of BCR-ABL1 (T315I). Another important mechanism is secondary chemotherapy resistance for different reasons. Therefore, it is necessary to study all the possible causes in our population, such as long-term adverse effects, resistance to TKIs, effects of other chemotherapy drugs and treatment noncompliance. In addition, a periodic follow-up of patients would be important to know the proportion of children with resistance to imatinib and to propose alternative treatments.

\section{KMT2A-AFF1}

The KMT2A-AFF1 $\mathrm{t}(4 ; 11)$ fusion was observed in this study in children between $4-16$ years of age. This frequency highlights that this rearrangement is not exclusive to infants with ALL, as has been previously reported [13]. Patients with ALL and the t(4;11) translocation have a poor prognosis [12]. In the present investigation, KMT2A-AFF1 patients had a poor prognosis with a high frequency of relapse, which was reflected in a low DFS (56\%) (Fig. 3).

Mexico is a country with limited financial resources where the detection of fusion rearrangements is not routinely performed in all hospitals due to high cost and lack of trained personnel in molecular biology. This assay could be used routinely to detect common fusion genes in children with ALL and may contribute significantly to clinical outcomes.

\section{Conclusions}

In conclusion, we developed a one-step RT-qPCR method that provides a sensitive and reliable assay for the rapid detection of four common fusion genes in pediatric patients with ALL. It could also provide less time to detect these alterations with cost savings. Moreover, the present study highlights the importance of the detection of common genetic fusions in Mexican children with ALL, taking into account that some have molecular subtypes associated with a poor prognosis. Mexico is a country with limited financial resources where the detection of fusion rearrangements is not routinely performed in all hospitals due to 
its high cost and the lack of trained personnel in molecular biology. This assay could be used routinely to detect common fusion genes in children with ALL and may contribute significantly to clinical outcomes.

\section{Declarations}

Funding: This work was funded by the Consejo Nacional de Ciencia y Tecnología (CONACyT): Grant numbers: PDCPN2013-01-215726, FIS/IMSS/PROT/1364; SALUD 2015-1-262190, FIS/IMSS/PROT/1533; CB-2015-1-258042, FIS/IMSS/PROT/1548, FONCICYT/37/2018, FIS/IMSS/PROT/1782, FORDECYTPRONACES/303019/2019, and FORDECYT-PRONACES/377883/2020; and by the Instituto Mexicano del Seguro Social: Grant numbers: FIS/IMSS/PROT/PRIO/14/031, FIS/IMSS/PROT/PRIO/15/048, FIS/IMSS/PROT/PRIO/18/080 and FIS/IMSS/PROT/PRIO/19/088. The funders had no role in study design, data collection and analysis, decision to publish, or preparation of the manuscript.

Acknowledgments: The authors acknowledge the Sequencing Laboratory, Instrument Center of National Medical Center "Siglo XXI", IMSS, Mexico City, for their technical assistance and support.

Conflicts of Interest: The authors declare no conflicts of interest.

\section{References}

1. Malouf C, Ottersbach K. Molecular processes involved in B cell acute lymphoblastic leukaemia. Cell Mol Life Sci. 2018;75(3):417-46.

2. Mejia-Arangure JM, et al. [Incidence of acute leukemia in children in Mexico City, from 1982 to 1991]. Salud Publica Mex. 2000;42(5):431-7.

3. Perez-Saldivar ML, et al. Childhood acute leukemias are frequent in Mexico City: descriptive epidemiology. BMC Cancer. 2011;11:355.

4. Pui $\mathrm{CH}$, et al. Biology, risk stratification, and therapy of pediatric acute leukemias: an update. J Clin Oncol. 2011;29(5):551-65.

5. Yoshihara $\mathrm{K}$, et al. The landscape and therapeutic relevance of cancer-associated transcript fusions. Oncogene. 2015;34(37):4845-54.

6. Stanulla M, Schrauder A. Bridging the gap between the north and south of the world: the case of treatment response in childhood acute lymphoblastic leukemia. Haematologica. 2009;94(6):748-52.

7. Andersen MT, et al. Characterization of $t(12 ; 21)$ breakpoint junctions in acute lymphoblastic leukemia. Leukemia. 2001;15(5):858-9.

8. Aplan PD, et al. Disruption of the human SCL locus by "illegitimate" V-(D)-J recombinase activity. Science. 1990;250(4986):1426-9.

9. Aplan PD. Causes of oncogenic chromosomal translocation. Trends Genet. 2006;22(1):46-55.

10. Mitelman F, Johansson B, Mertens F. The impact of translocations and gene fusions on cancer causation. Nat Rev Cancer. 2007;7(4):233-45. 
11. Wiemels JL, et al. Prenatal origin of acute lymphoblastic leukaemia in children. Lancet. 1999;354(9189):1499-503.

12. Mori $\mathrm{H}$, et al. Chromosome translocations and covert leukemic clones are generated during normal fetal development. Proc Natl Acad Sci U S A. 2002;99(12):8242-7.

13. Meyer C, et al. The MLL recombinome of acute leukemias in 2017. Leukemia. 2018;32(2):273-84.

14. Paulsson K, et al., Characterisation of genomic translocation breakpoints and identification of an alternative TCF3/PBX1 fusion transcript in $t(1 ; 19)(q 23 ; p 13)$-positive acute lymphoblastic leukaemias. Br J Haematol, 2007. 138(2): p. 196-201.

15. Le Scouarnec S, Gribble SM. Characterising chromosome rearrangements: recent technical advances in molecular cytogenetics. Heredity. 2012;108(1):75-85.

16. Pakakasama $S$, et al. Simple multiplex RT-PCR for identifying common fusion transcripts in childhood acute leukemia. Int J Lab Hematol. 2008;30(4):286-91.

17. Slack JL, et al. Pre-clinical validation of a novel, highly sensitive assay to detect PML-RARalpha mRNA using real-time reverse-transcription polymerase chain reaction. J Mol Diagn. 2001;3(4):1419.

18. Cerchione C, Locatelli F, Martinelli G. Dasatinib in the Management of Pediatric Patients With Philadelphia Chromosome-Positive Acute Lymphoblastic Leukemia. Front Oncol. 2021;11:632231.

19. Jimenez-Morales S, et al. BCR-ABL, ETV6-RUNX1 and E2A-PBX1: prevalence of the most common acute lymphoblastic leukemia fusion genes in Mexican patients. Leuk Res. 2008;32(10):1518-22.

20. Siraj $A K$, et al. Preclinical validation of a monochrome real-time multiplex assay for translocations in childhood acute lymphoblastic leukemia. Clin Cancer Res. 2002;8(12):3832-40.

21. Ariffin $\mathrm{H}$, et al. Validation of a multiplex RT-PCR assay for screening significant oncogene fusion transcripts in children with acute lymphoblastic leukaemia. Singapore Med J. 2003;44(10):517-20.

22. Bekker-Mendez VC, et al. Prevalence of gene rearrangements in Mexican children with acute lymphoblastic leukemia: a population study-report from the Mexican Interinstitutional Group for the identification of the causes of childhood leukemia. Biomed Res Int. 2014;2014:210560.

23. Juarez-Avendano G, et al. Poor Prognosis Biomolecular Factors Are Highly Frequent in Childhood Acute Leukemias From Oaxaca, Mexico. Technol Cancer Res Treat. 2020;19:1533033820928436.

24. Borkhardt A, et al. Incidence and clinical relevance of TEL/AML1 fusion genes in children with acute lymphoblastic leukemia enrolled in the German and Italian multicenter therapy trials. Associazione Italiana Ematologia Oncologia Pediatrica and the Berlin-Frankfurt-Munster Study Group. Blood. 1997;90(2):571-7.

25. Amor DJ, et al. High frequency of $t(12 ; 21)$ in childhood acute lymphoblastic leukemia detected by RTPCR. Pathology. 1998;30(4):381-5.

26. McLean TW, et al. TEL/AML-1 dimerizes and is associated with a favorable outcome in childhood acute lymphoblastic leukemia. Blood. 1996;88(11):4252-8. 
27. Perez-Vera P, et al. Analysis of gene rearrangements using a fluorescence in situ hybridization method in Mexican patients with acute lymphoblastic leukemia: experience at a single institution. Cancer Genet Cytogenet. 2008;184(2):94-8.

28. Perez-Vera P, et al. Detection of ETV6 and RUNX1 gene rearrangements using fluorescence in situ hybridization in Mexican patients with acute lymphoblastic leukemia: experience at a single institution. Cancer Genet Cytogenet. 2005;162(2):140-5.

29. Martinez-Mancilla $M$, et al. Clinical relevance of the fusion transcripts distribution pattern in mexican children with acute lymphoblastic leukemia. J Pediatr Hematol Oncol. 2013;35(3):170-3.

30. Carranza C, et al. Frequency of the ETV6-RUNX1, BCR-ABL1, TCF3-PBX1, and MLL-AFF1 fusion genes in Guatemalan pediatric acute lymphoblastic leukemia patients and their ethnic associations. Cancer Genet. 2013;206(6):227-32.

31. Aldrich MC, et al. Cytogenetics of Hispanic and White children with acute lymphoblastic leukemia in California. Cancer Epidemiol Biomarkers Prev. 2006;15(3):578-81.

32. Walsh KM, et al. Genomic ancestry and somatic alterations correlate with age at diagnosis in Hispanic children with B-cell acute lymphoblastic leukemia. Am J Hematol. 2014;89(7):721-5.

33. Kumar $S$, et al. Identifying fusion transcripts using next generation sequencing. Wiley Interdiscip Rev RNA. 2016;7(6):811-23.

34. Mata-Rocha M, et al., Identification and Characterization of Novel Fusion Genes with Potential Clinical Applications in Mexican Children with Acute Lymphoblastic Leukemia. Int J Mol Sci, 2019. 20(10).

35. Wiemels JL, et al. Site-specific translocation and evidence of postnatal origin of the t(1;19) E2A-PBX1 fusion in childhood acute lymphoblastic leukemia. Proc Natl Acad Sci U S A. 2002;99(23):15101-6.

36. Aspland SE, Bendall HH, Murre C. The role of E2A-PBX1 in leukemogenesis. Oncogene. 2001;20(40):5708-17.

37. Baker SJ, Reddy EP. B cell differentiation: role of E2A and Pax5/BSAP transcription factors. Oncogene. 1995;11(3):413-26.

38. Jeha S, et al., Increased risk for CNS relapse in pre-B cell leukemia with the t(1;19)/TCF3-PBX1. Leukemia, 2009. 23(8): p. 1406-9.

39. Nunez-Enriquez JC, Mejia-Arangure JM. [Molecular biology and childhood leukemia: E2A-PBX1 and central nervous system relapse]. Rev Med Inst Mex Seguro Soc. 2015;53(Suppl 3):S236-9.

40. Kager $L$, et al. Incidence and outcome of TCF3-PBX1-positive acute lymphoblastic leukemia in Austrian children. Haematologica. 2007;92(11):1561-4.

41. Felice MS, et al. Prognostic impact of $t(1 ; 19) /$ TCF3-PBX1 in childhood acute lymphoblastic leukemia in the context of Berlin-Frankfurt-Munster-based protocols. Leuk Lymphoma. 2011;52(7):1215-21.

42. Hu Y, et al. E2A-PBX1 exhibited a promising prognosis in pediatric acute lymphoblastic leukemia treated with the CCLG-ALL2008 protocol. Onco Targets Ther. 2016;9:7219-25. 
43. Bernt KM, Hunger SP. Current concepts in pediatric Philadelphia chromosome-positive acute lymphoblastic leukemia. Front Oncol. 2014;4:54.

44. Lee HJ, et al. Philadelphia chromosome-positive acute lymphoblastic leukemia: current treatment and future perspectives. Cancer. 2011;117(8):1583-94.

\section{Figures}

A

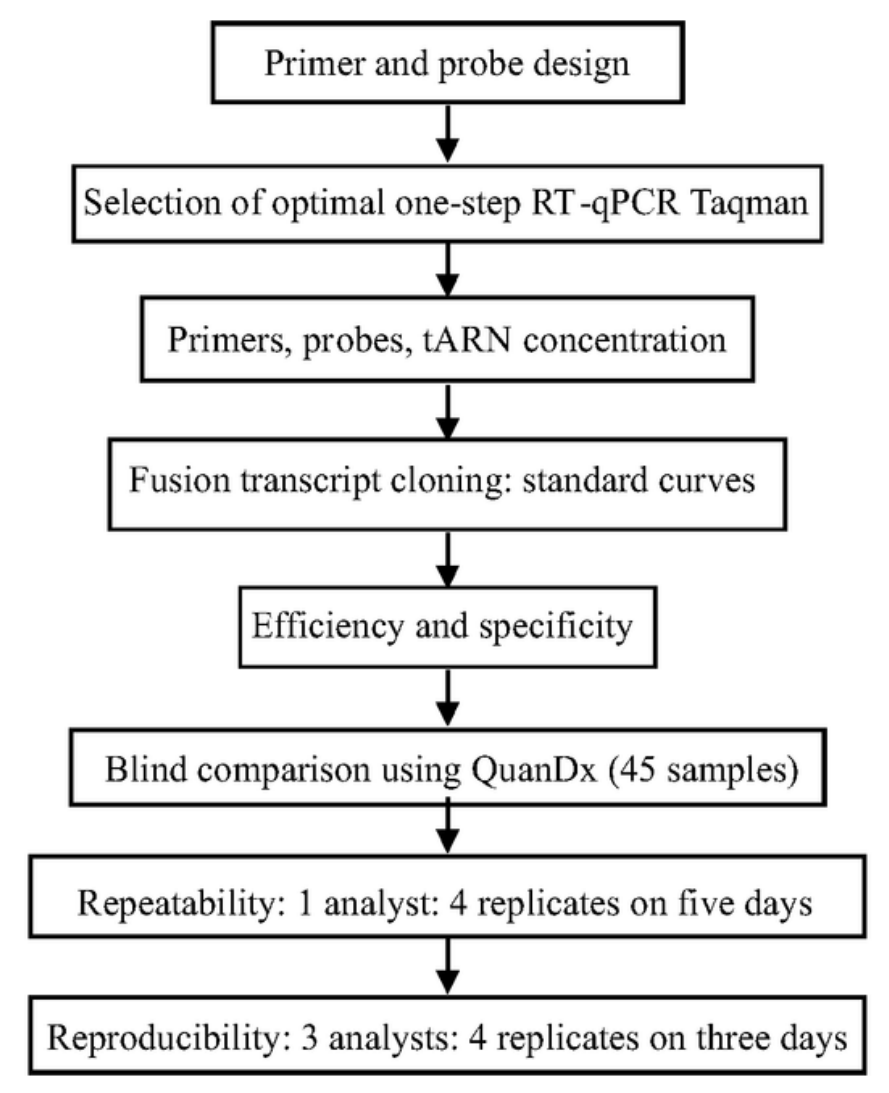

B Analysis of clinical samples

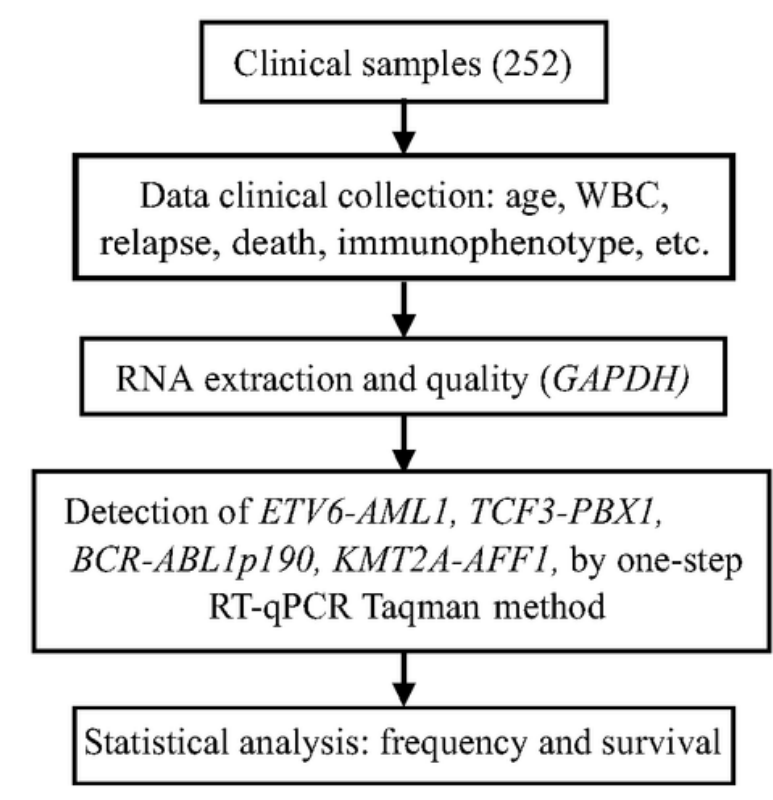

\section{Figure 1}

Schematic diagram showing the research workflow. The two main stages of research were A) development of the method (design, concentration optimization, efficiency, repeatability, reproducibility) and B) analysis of clinical samples (sample validation, RT-qPCR assay, data collection and data analysis). 
A

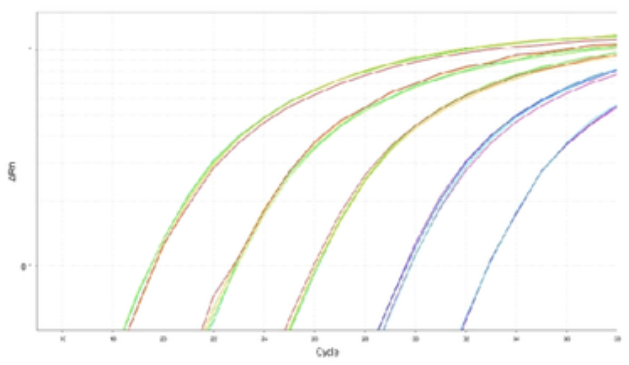

B

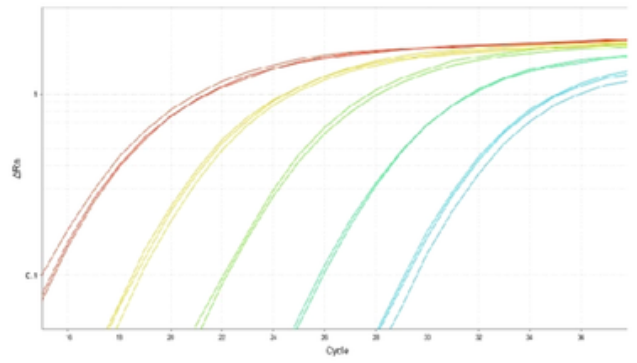

C

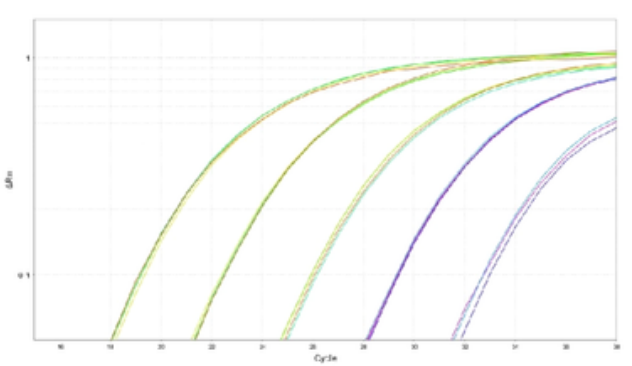

$\mathbf{D}$

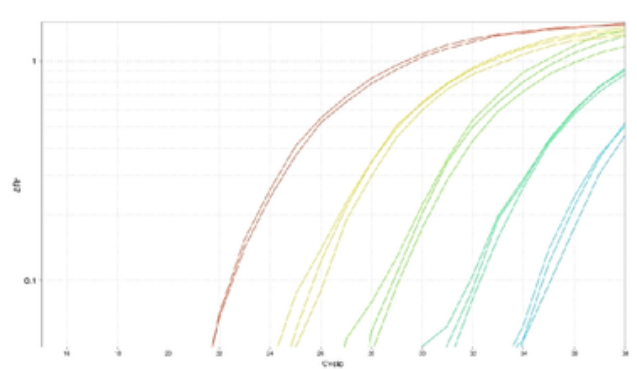

$\mathbf{E}$

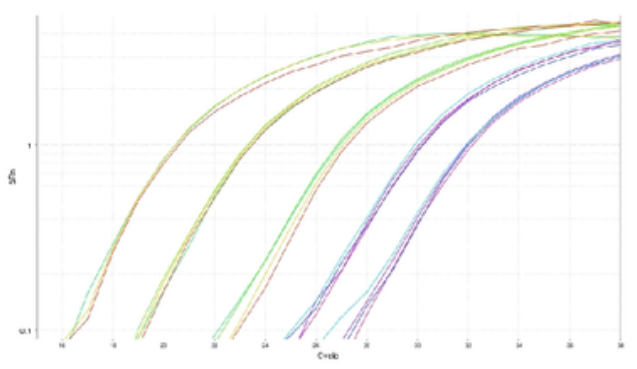

ETV6-RUNX1

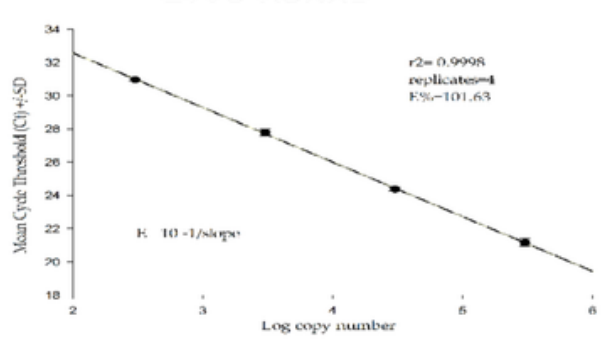

TCF3-PBX1
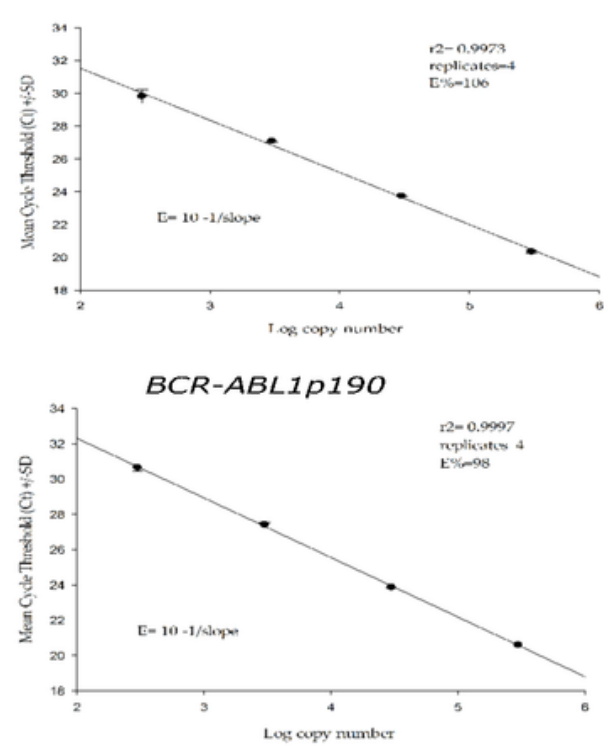

KMT2A-AFF1

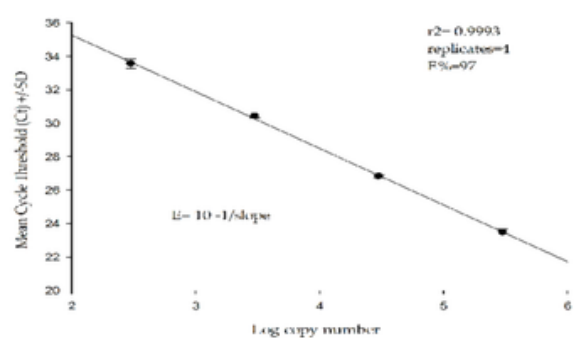

GAPDH

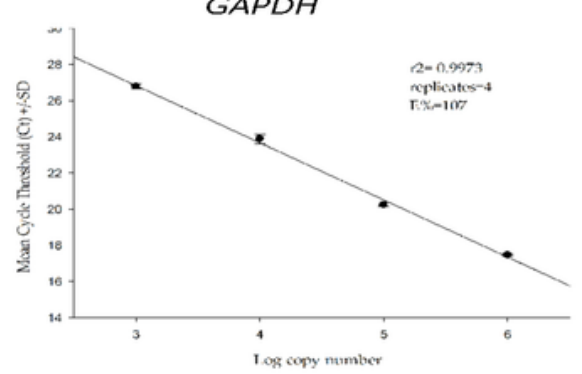

\section{Figure 2}

Linear dynamic range of one-step RT-qPCR using plasmid DNA. The standards used in this study were well-characterized plasmid DNA dilutions that had been directly quantitated using a Qubit fluorometer. The standard curve for real-time RT-PCR was generated from the Ct values plotted against 10-fold serial dilutions (4×106 to $4 \times 102$ copies). Triplicate samples were used for the assay. Amplification plot and standard curve of ETV6-RUNX1 (A), TCF3-PBX1 (B), BCR-ABL1p190 (C), KMT2A-AFF1 (D) and GAPDH (E). 

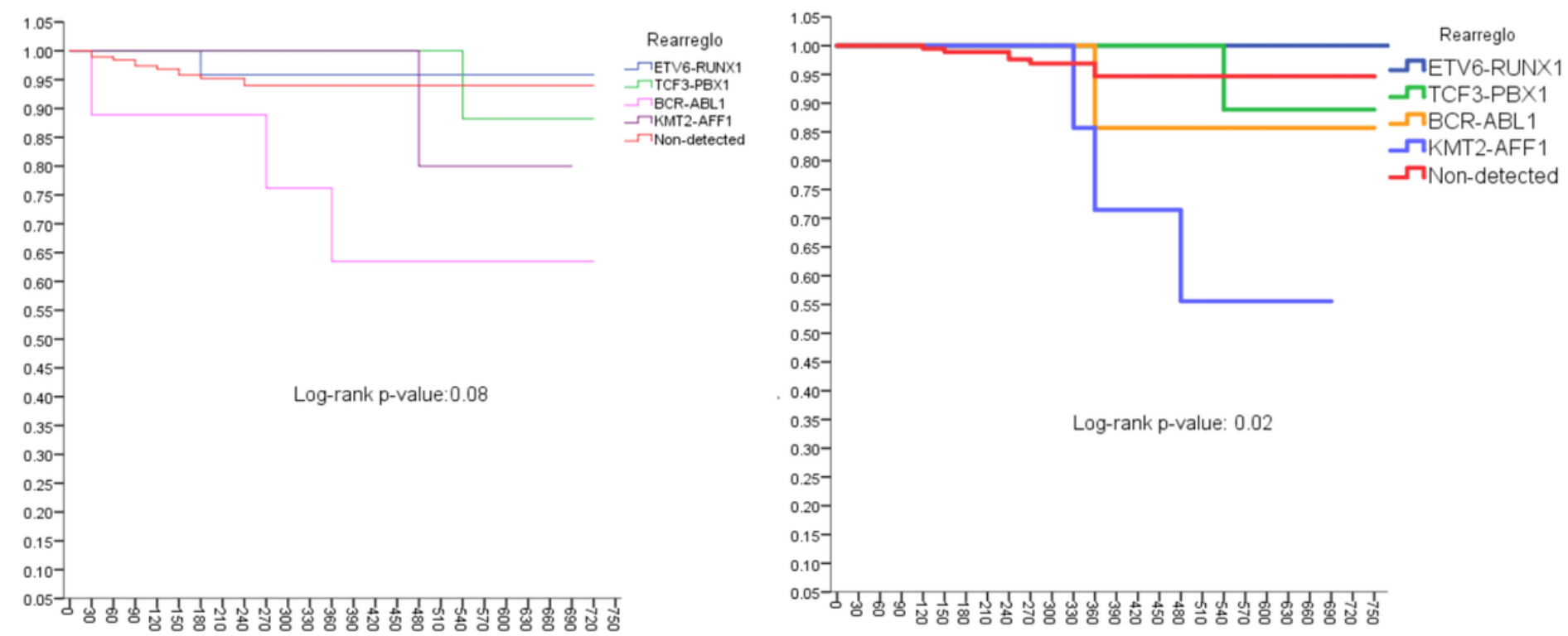

\section{Figure 3}

Overall survival (OS) and disease-free survival (DFS) rates for childhood with ALL by each fusion gene. Outcome was significantly better in patients with ETV6-RUNX1, TCF3-PBX1 and nondetected fusion gene transcripts than in those with rearranged KMT2A-AFF1. DFS was $100,97,89,86$, and $56 \%$ and overall survival (OS) was $96,94,88,63$, and $80 \%$ for ETV6-RUNX1, no fusion gene transcripts detected, E2APBX1, BCR-ABL1p190, and KMT2A-AFF1, respectively.

\section{Supplementary Files}

This is a list of supplementary files associated with this preprint. Click to download.

- SupplementaryFigure1.pdf

- SupplementaryFigure2.pdf

- SupplementaryFigure3.pdf

- SupplementaryTable1.xlsx 